\title{
ADAMTS1 and HSPG2 mRNA levels in cumulus cells are related to human oocyte quality and controlled ovarian hyperstimulation outcomes
}

\author{
Yerong Ma ${ }^{1,2} \cdot$ Jiamin Jin ${ }^{1,2} \cdot$ Xiaomei Tong ${ }^{1,2} \cdot$ Weijie Yang ${ }^{1,2} \cdot$ Peipei Ren ${ }^{1,2} \cdot$ Yongdong Dai $^{1,2} \cdot$ Yibin Pan $^{1,2}$. \\ YinLi Zhang ${ }^{1,2}$. Songying Zhang ${ }^{1,2}$ (i)
}

Received: 15 August 2019 / Accepted: 12 December 2019 / Published online: 23 January 2020

(C) The Author(s) 2020

\begin{abstract}
Purpose The study investigated potential correlations between the expression levels of ADAMTS1 and HSPG2 in cumulus cells (CCs) and controlled ovarian hyperstimulation $(\mathrm{COH})$ outcomes.

Methods RT-PCR was used to determine ADAMTS1 and HSPG2 mRNA levels in mice CCs at different timepoints $(0,4,8,12$, and $16 \mathrm{~h}$ ) after human chorionic gonadotropin (hCG) injection, and in CCs after RNAi treatment. Women with polycystic ovary syndrome (PCOS) $(n=45)$ and normal ovulatory controls $(n=103)$ undergoing IVF/ICSI were recruited. Relative ADAMTSI and $H S P G 2$ mRNA levels were measured by RT-PCR. Moreover, correlations of ADAMTS1 and HSPG2 levels with COH outcomes were analyzed.

Results At different timepoints after hCG treatment, ADAMTS1 mRNA had the highest level at $12 \mathrm{~h}$, whereas HSPG2 showed opposite profiles to ADAMTS1 with the lowest level at $12 \mathrm{~h}$. HSPG2 expression was upregulated after ADAMTS1 RNAi treatment The PCOS group had higher HSPG2 and lower ADAMTS1 expression levels than controls. In normal ovulatory women (control group), a higher expression of ADAMTS1 and lower expression of HSPG2 were associated with more mature oocytes, transplantable embryos, and good quality embryos, whereas higher transplantable embryo rates and good quality embryo rates were obtained only with lower HSPG2 expression. ROC curves showed the co-measurement of ADAMTS1 and HSPG2 had a better predictive power than separate analyses.

Conclusion The dynamic profiles of ADAMTS1 and HSPG2 were inversely correlated in CCs. In PCOS and normal ovulatory patients, higher ADAMTS1 and lower HSPG2 expression levels in CCs were related to better $\mathrm{COH}$ outcomes.
\end{abstract}

Keywords ADAMTS1 $\cdot$ HSPG2 $\cdot$ Cumulus cells $\cdot$ PCOS $\cdot \mathrm{COH}$

Yerong Ma and Jiamin Jin contributed equally to this work.

Electronic supplementary material The online version of this article (https://doi.org/10.1007/s10815-019-01659-8) contains supplementary material, which is available to authorized users.

YinLi Zhang

zhangyinli@zju.edu.cn

Songying Zhang

zhangsongying@zju.edu.cn

1 Assisted Reproduction Unit, Department of Obstetrics and Gynecology, Sir Run Run Shaw Hospital, School of Medicine, Zhejiang University, No. 3 Qingchun East Road, Jianggan District, Hangzhou 310016, China

2 Key Laboratory of Reproductive Dysfunction Management of Zhejiang Province, No. 3 Qingchun East Road, Jianggan District, Hangzhou 310016, China

\section{Introduction}

Ovulation, stimulated by a luteinizing hormone (LH) surge, is a dynamic series of events that includes oocyte meiosis resumption, cumulus expansion, follicle rupture, and cumulus oocyte complex (COC) release. LH mainly triggers the ERK1/ ERK2 signaling cascade in granulosa cells (GCs) to induce the expression of many genes crucial for ovulation, oocyte meiosis, and extracellular matrix (ECM) remodeling [1]. Many of these ERK1/ERK2-dependent genes, including AREG, ADAMTS1, and CITED2, in GCs were associated with oocyte quality and IVF outcomes [2-7].

ECM remodeling is characterized by the degradation of localized ECM components, including versican, laminin, collagen IV, perlecan, and fibulin [2, 8-12]. ECM remodeling occurs throughout the whole ovulation process. For example, 
cumulus cell (CCs) expansion includes hyaluronan-rich ECM after an LH surge. Furthermore, successful ovulation requires precise ECM remodeling to ensure follicle rupture [11, 13-16].

ADAMTS1 (a disintegrin and metalloprotease with thrombospondin type 1 motif 1 ), a member of the proteinase family, has catalytic activity against proteoglycans (such as aggrecan and versican) to remodel the ECM $[2,8,14$, 17-22]. Using an ADAMTS1 knockout mouse model, it was convincingly shown that ADAMTS1 plays key roles in female reproduction [3]. ADAMTS1 null mice have morphologically abnormal ovaries, reduced numbers of ovulated oocytes, and a reduced fertilization rate. Compared with ADAMTS1 ${ }^{ \pm}$mice, ADAMTS1 $1^{-/}$mice have high levels of versican, which is undetectable in $A D A M T S I^{ \pm}$ovaries, indicating that the processing of versican by ADAMTS1 is involved in ovulating follicle remodeling [14]. This indicates ADAMTS1, and versican levels are closely associated with oocyte number and competence. In recent years, several investigators have demonstrated a close correlation between ADAMTS1 levels in human CCs or follicle fluids and impaired oocyte quality in polycystic ovary syndrome (PCOS) patients [23-26]. Consistent with ADAMTS1 $1^{-/-}$mice, ADAMTS1 levels in CCs were decreased in PCOS patients, which might contribute to abnormalities, such as low fertilization rate and cleavage rate $[4,7,26]$. To date, no study has evaluated the relationship between ADAMTS1 level and controlled ovarian hyperstimulation $(\mathrm{COH})$ outcomes in normal ovulatory patients.

Perlecan/heparan sulfate proteoglycan 2 (HSPG2) encodes perlecan, a substrate highly expressed in ovarian ECM that is potentially cleaved by ADAMTS1 [27]. Perlecan is an extracellular proteoglycan involved in tumor angiogenesis, proliferation, and invasion. It is also a potential ECM substrate of ADAMTS1 during ovulation [27-29] and is involved in the stabilization of other molecules, permeability of the glomerulus to macromolecules, and cell adhesion [8,30-33]. It is a potent inhibitor of smooth muscle cell proliferation and is therefore thought to help maintain vascular homeostasis [32, 34-36]. It also promotes growth factor activity, thereby stimulating endothelial growth and regeneration [13, 32, 35, 37]. There have been limited studies regarding the function of $H S P G 2$ in reproduction. One study showed that the quantity of perlecan in follicular fluid with fertilized oocytes was significantly greater than that with non-fertilized oocytes from the same patient [38]. Furthermore, low levels of perlecan were observed in PCOS patient follicular fluid [38]. However, whether HSPG2 mRNA levels in human CCs are associated with oocyte quality is unknown. Thus, we investigated whether the mRNA levels of ADAMTS1 and HSPG2 in human CCs indicated oocyte quality.

In this study, we investigated the timing of the expression of ADAMTS1 and HSPG2 during ovulation by RT-PCR using mouse CCs following hCG injection. We found the opposite expression pattern of ADAMTS1 and HSPG2 at the mRNA level during ovulation. To determine the effect of ADAMTS1 on HSPG2 expression, we knocked down ADAMTS1 in mouse COCs. We found that ADAMTSI RNAi increased $H S P G 2$ expression in CCs. Thus, we investigated whether ADAMTS1 and HSPG2 mRNA levels were related to $\mathrm{COH}$ outcomes. Therefore, we performed RT-PCR to compare ADAMTS1 and HSPG 2 mRNA levels between PCOS patients and normal ovulatory women and confirmed the inverse pattern and predictive power of the two molecules. Last, in normal ovulatory women, the correlations between ADAMTS1 or HSPG 2 mRNA level and $\mathrm{COH}$ outcomes (oocyte number, oocyte quality, and embryo developmental potential) were intensively investigated.

\section{Materials and methods}

\section{Mouse mural GCs and CCs collection}

C57BL/6 mice were obtained from the Zhejiang Academy of Medical Science, China. Animal care and experimental procedures were conducted in accordance with the Animal Research Committee guidelines of Zhejiang University.

Female wild-type C57BL/6 mice at postnatal day (PD) 23 were primed with $5.0 \mathrm{IU}$ pregnant mare serum gonadotrophin (PMSG). And $48 \mathrm{~h}$ later, mice were injected with human chorionic gonadotropin ( $\mathrm{hCG}$ ) for different treatment times $(0,4$, 8,12 , and $16 \mathrm{~h}$ ). At these timepoints, ovaries were dissected, and follicles were punctured using needles. Then, mural GCs were harvested directly and CCs were obtained by removing the oocytes mechanically from COCs.

\section{Mouse COCs collection, RNAi, and in vitro maturation (IVM)}

Enclosed COCs were collected by puncturing the ovaries from mice with 5 IU PMSG treatment for $48 \mathrm{~h}$. IVM medium (Easy Check, China, M2115) with $2.5 \mu \mathrm{M}$ milrinone (MCE, USA, HY-14252) was used to inhibit CCs expansion during collection and culture with siRNA. Prior to COCs collection, specific siRNAs (5'-GGAAGTACTGTGAAGGCAA-3') (RIBOBIO, China, S181130134515) for the mouse ADAMTS1 gene and a negative control at a final concentration of $50 \mathrm{pmol} / \mathrm{ml}$ were mixed with Lipofectamine ${ }^{\mathrm{TM}} 3000$ Transfection Reagent (Thermo Fisher Scientific, USA, L3000015) for $30 \mathrm{~min}$ and then added into the cultured IVM medium containing $2.5 \mu \mathrm{M}$ milrinone. The COCs were transferred to the RNAi medium and cultured for $24 \mathrm{~h}$ in a $5 \% \mathrm{CO}_{2}$ incubator at $37^{\circ} \mathrm{C}$. After transfection with siRNA or negative control for $24 \mathrm{~h}$, COCs were transferred and allowed to resume meiosis in new fresh IVM medium (Easy Check, China, 
$\mathrm{M} 2115$ ) without milrinone and then cultured in a $5 \% \mathrm{CO}_{2}$ incubator at $37^{\circ} \mathrm{C}$ for $12 \mathrm{~h}$. The CCs were harvested from 10 matured COCs by a mechanical method for RNA isolation.

\section{Patient selection}

This study included 148 women undergoing IVF/ICSI because of PCOS ( $n=45$; ICSI, $n=5$; IVF, $n=40$ ) or tubal factor or male factor ( $n=103$, ICSI, $n=23$; IVF, $n=80)$ at the Reproductive Center of Sir Run Run Shaw Hospital affiliated to Zhejiang University from July 2017 to June 2018. The experiment was certified by the ethics committee of Sir Run Run Shaw Hospital affiliated to Zhejiang University (20190215-2). All patients signed informed consent regarding the collection of CCs.

Of all patients undergoing IVF/ICSI, we selected patients who were between 20 and 35 years old. Our study did not affect clinical treatment options, but we only chose patients who were treated with a long protocol. Briefly, mid-luteal gonadotropin-releasing hormone ( $\mathrm{GnRH})$ agonist (Ferring) and ovarian stimulation with a daily subcutaneous dose of hMG (Menogon, Ferring) were started on the third day of the menstrual cycle. When the leading follicles reached $18 \mathrm{~mm}$ in diameter, women received hCG (Merck, Serono). Patients with a minimum of seven large follicles $\geq 14 \mathrm{~mm}$ at the final ultrasound before oocyte retrieval were invited to attend.

At the same time, patients who did not meet the follow-up criteria were excluded. Inclusion criteria for normal ovulatory women $(n=103)$ were age $>20$ and $<35$ years old, regular menstrual periods between 23 and 35 days, and IVF/ICSI indication for tubal or male factor. Patients were excluded for any of the following conditions: (1) abnormal endocrinology, such as polycystic ovary syndrome or premature ovary failure; (2) day 3 serum FSH level > 10 IU/l; (3) ovarian hyperstimulation syndrome (OHSS); or (4) suffering from other diseases (cardiovascular, pulmonary, liver, or kidney disease).

Based on the Rotterdam criteria [39], patients with PCOS and two of the following criteria were included: (1) rare ovulation or anovulation; (2) hyperandrogenism or clinical manifestations of hyperandrogenism (such as hairy, acne); and (3) polycystic ovarian changes. Patients with other causes of hyperandrogenism (such as hyperprolactinemia and thyroid diseases, congenital adrenal cortical hyperplasia, Cushing's syndrome, androgen-secreting tumors, 21-hydroxylase deficiency atypical adrenal cortical hyperplasia, and exogenous androgen use) were excluded.

\section{Human CCs collection}

Aspiration of the oocytes was performed transvaginally by ultrasound guidance $36 \mathrm{~h}$ after hCG administration. Immediately after the isolation of COCs, professional staff cut part of the cumulus mass off. CCs from all $18-20 \mathrm{~mm}$ follicles of a patient were pooled. Erythrocytes were removed by adding erythrocyte lysis buffer (EL-buffer, Qiagen, Germany). Then, CCs were washed with $1 \mathrm{ml}$ phosphatebuffered saline (PBS) twice and centrifuged for $10 \mathrm{~min}$ at $800 \mathrm{x} \mathrm{g}$ to form a pellet. The pellet was collected and stored at $-80{ }^{\circ} \mathrm{C}$ until RNA extraction.

\section{Human mural GCs collection}

Human mural GCs were isolated from the follicular fluid by a pipette, and erythrocyte lysis buffer was added to remove erythrocytes. Similar to CCs, mural GCs were washed with PBS twice and centrifuged for $10 \mathrm{~min}$ at $800 \mathrm{x}$ g to form a pellet. The pellet was collected and stored at $-80{ }^{\circ} \mathrm{C}$ until RNA extraction. Mural GCs from all 18-20 mm follicles of a patient were pooled.

\section{RNA isolation, reverse transcription, and RT-PCR}

Total RNA was isolated using the RNeasy Plus Micro Kit (Qiagen, Germany, 74,034) according to the manufacturer's protocol. Then, RNA concentration was determined by NanoDrop 2000 (Thermo Fisher) and cDNA was obtained from total RNA ( $50 \mathrm{ng}$ ) of each sample by reverse transcription using oligo $(\mathrm{dT})_{15}$ and reverse transcriptase (Promega, USA, A3500), according to the manufacturer's instructions, at $42{ }^{\circ} \mathrm{C}$ for $15 \mathrm{~min}$, followed by $95^{\circ} \mathrm{C}$ for $5 \mathrm{~min}$ in $20 \mu \mathrm{l}$ total volume.

Quantitative real-time PCR was performed using SYBR Green Master Mix (ABI, Germany, DBI-2044) in an Applied Biosystems 7500 Real-Time PCR System. An aliquot (10\%) of cDNA was subjected to 40 amplification cycles of PCR with the primers listed in Supplementary Table 1. Cycling parameters were one cycle for $2 \mathrm{~min}$ at $50^{\circ} \mathrm{C}$, one cycle for $10 \mathrm{~min}$ at $95{ }^{\circ} \mathrm{C}, 40$ cycles at $95{ }^{\circ} \mathrm{C}$ for $15 \mathrm{~s}$, and $60{ }^{\circ} \mathrm{C}$ for $20 \mathrm{~s}$. For each experiment, three replicates were included in each qPCR reaction. We performed melting curve analysis at the end of each run to ensure a single amplicon.

The relative levels of endogenous $\beta$-actin mRNA (ACTB) mRNA were used as an internal control [40], and data were analyzed using the $2^{-(\Delta \Delta \mathrm{Ct})}$ method. Only samples whose cycle threshold $(\mathrm{Ct})$ values of ACTB were between 21 and 22 were included in the analysis.

\section{Assessment of oocyte and embryo quality}

Regarding clinical outcomes, the development conditions of oocytes and embryos were observed and recorded by professional staff. MII oocytes with their corresponding polar bodies and fertilized oocytes with pronuclei were observed at 4-6 h and 16-18 h after insemination, respectively. Good quality embryos and transferable embryos were judged according to 
a cleavage embryo scoring system [41] at day 3. Professional staff observed the number of cleavage spheres, their symmetry, cytoplasmic morphology, and the number of fragments produced during division. According to these parameters, the embryos were divided into four grades. Grade I-II embryos have equally sized blastomeres with $0 \%-20 \%$ fragmentation. Grade III embryos have unequal sizes and 20\% fragmentation. Grade IV embryos have low developmental potential with an abnormal appearance. The grade I-II embryos were recorded as good quality embryos, and grade I-III embryos were recorded as transferable embryos. Only transferable embryos were frozen on day 3, after the assessment of embryo quality. Concurrently, grade IV embryos were discarded. When the patient had reached the condition of embryo transfer, two embryos were thawed and transferred, including endometrial thickness between 8 and $12 \mathrm{~mm}, \mathrm{E} 2>100 \mathrm{IU} / \mathrm{L}$, and $\mathrm{LH}<10 \mathrm{IU} / \mathrm{L}$. High-level embryos were transferred as a priority. For implantation, we included only the first transfer from each patient, and all embryos transferred were vitrifiedwarmed.

The fertilization rate of IVF $=$ the number of zygotes $/$ the total number of retrieved oocytes $\times 100 \%$.

The fertilization rate of ICSI $=$ the number of zygotes $/$ the number of MII stage oocytes $\times 100 \%$.

The transplantable embryo rate $=$ the number of transferable embryos (grade I-III) / the number of total embryos $\times$ $100 \%$.

The good quality embryo rate $=$ the number of good quality embryos (grade I-II) / the number of total embryos $\times 100 \%$.

\section{Clinical data collection}

The research team was blinded to experimental data until all clinical data had been collected from all patients. The clinical staff obtained the clinical data, including the number of oocytes retrieved, MII-stage oocytes, good quality embryos, transferable embryos, good quality embryos rate, transferable rate, and implantation outcome.

\section{Statistical analysis}

All analyses were performed using SPSS software version 23.0 (SPSS Inc. Chicago, IL, USA). Tests of statistical significance were two sided and considered significant when $P<0.05$. For the relationship between ADAMTS1 or HSPG2 mRNA level and $\mathrm{COH}$ outcomes, the Kolmogorov-Smirnov test was used to judge whether parameters showed continuous distribution. If so, unpaired Student's two-tailed $t$-tests were applied. If not, non-parametric tests were applied. Analyses using non-parametric tests were marked with \# in Tables 1,2, 3. The mean \pm standard error (SE) was used for the descriptive statistics of data. To determine whether ADAMTS1 and $H S P G 2$ mRNA levels were associated with successful implantation, receiver operating characteristic (ROC) analyses were used to determine the predictive potential of ADAMTS1 and $H S P G 2$ mRNA level on implantation. Based on the ROC curve, positive and negative predict values were calculated on the Youden index cut-off point.

\section{Results}

\section{The dynamics of ADAMTS1 and HSPG2 mRNA levels during ovulation}

We wished to evaluate the expression timing of ADAMTS1 and $H S P G 2$ during ovulation and therefore conducted experiments in the mouse evaluating the expression of these genes following hCG injection. Human oocytes were collected at $36 \mathrm{~h}$ after the injection of hCG in the $\mathrm{COH}$ cycle, and this timepoint was equivalent to approximately $12 \mathrm{~h}$ in mice. The expression levels of ADAMTS1 or HSPG2 were comparable between mural GCs and CCs at hCG $12 \mathrm{~h}$ in mice or $36 \mathrm{~h}$ in humans (Fig. 1A-D). To evaluate the timing of the expression of ADAMTS1 and HSPG2 during ovulation, mouse ovarian $\mathrm{CCs}$ at different timepoints were collected after hCG treatment for RT-PCR. As shown in Fig. 1E, ADAMTS1 mRNA levels were low before $8 \mathrm{~h}$, sharply increased at $12 \mathrm{~h}$, and then decreased to the basal level at $16 \mathrm{~h}$, at which time the level at hCG $12 \mathrm{~h}$ was over tenfold higher compared with $0 \mathrm{~h}$. In contrast to ADAMTS1, the mRNA level of HSPG2 in mouse CCs decreased first and then increased, with the lowest expression at $12 \mathrm{~h}$ after hCG injection (Fig. 1F). This result indicates that the dynamic expression profiles of ADAMTS1 and $H S P G 2$ mRNA have an inverse relationship.

\section{The relative expression of $H S P G 2$ increases after disturbing ADAMTS1 expression in COCs}

We wished to determine the effect of ADAMTS1 expression on $H S P G 2$ expression and therefore conduced RNAi experiments knocking down ADAMTS1 expression in the mouse during in vitro maturation, and CCs were collected for RTPCR. When ADAMTS1 mRNA was depleted in mouse CCs, the relative $H S P G 2$ mRNA level was significantly increased (Fig. 1G). This result indicates the essential role of ADAMTS1 to regulate $H S P G 2$ mRNA levels during ovulation.

\section{Lower ADAMTS1 and higher HSPG2 mRNA levels in CCs from PCOS patients compared with normal ovulatory patients}

To explore the relationship between the expression of ADAMTS1 and HSPG2 and COH outcomes, we compared the mRNA levels of ADAMTS1 and HSPG2 in the control group with tubal or male factor patients $(n=103)$ and the 
Table 1 Patients' characteristics of PCOS patients and normal ovulatory women

\begin{tabular}{lccc}
\hline Variable & PCOS women $(N=45)$ & Normal ovulatory women $(N=103)$ & $P$ value \\
\hline Age (years) & $28.796 \pm 0.073$ & $29.544 \pm 0.034$ & NS \\
BMI $\left(\mathrm{Kg} / \mathrm{m}^{2}\right)$ & $21.379 \pm 0.056$ & $21.776 \pm 0.025$ & $\mathrm{NS}$ \\
Basal FSH (IU/L) & $6.120 \pm 0.038$ & $6.270 \pm 20.020$ & $\mathrm{NS}$ \\
LH (IU/L) & $8.227 \pm 0.096$ & $5.015 \pm 0.056$ & 0.0104 \\
Serum AMH (ng/mL) & $10.226 \pm 0.105$ & $4.818 \pm 0.022$ & $<0.0001$ \\
hCG dose (IU) & $6130.953 \pm 29.977$ & $6868.687 \pm 12.752$ & 0.0009 \\
\hline
\end{tabular}

Continuous variables are expressed as mean \pm SE. NS, no significance; PCOS, polycystic ovarian syndrome; BMI, body mass index; FSH, follicle stimulating hormone; LH, luteinizing hormone; $\mathrm{AMH}$, anti-Müllerian hormone
PCOS group $(n=45)$. Basal information of the paired groups is shown in Table 1. Patients were between 20 and 35 years old (mean \pm SE: $28.796 \pm 0.073$ years in the PCOS group vs. $29.544 \pm 0.034$ years in the control group, NS). The body mass index (BMI) of subjects was between 18 and $26 \mathrm{~kg} / \mathrm{m}^{2}$ in both groups (mean \pm SE: $21.379 \pm 0.056 \mathrm{~kg} / \mathrm{m}^{2}$ in the PCOS group vs. $21.776 \pm 0.025 \mathrm{~kg} / \mathrm{m}^{2}$ in the control group, $\mathrm{NS}$ ). Basal FSH was within the normal range (mean \pm SE: $6.120 \pm 0.038 \mathrm{IU} / \mathrm{L}$ in the PCOS group vs. $6.270 \pm 0.194 \mathrm{IU} /$ $\mathrm{L}$ in the control group, NS), whereas basal LH level in the PCOS group was nearly 1.6 -fold higher than that in the control group (mean $\pm \mathrm{SE}: 8.227 \pm 0.096 \mathrm{IU} / \mathrm{L}$ vs. $5.015 \pm$ $0.056 \mathrm{IU} / \mathrm{L}, P=0.0104)$. Human anti-Müllerian hormone $(\mathrm{AMH})$ in the PCOS group was over twofold higher compared with the control group (mean \pm SE: $10.226 \pm 0.105 \mathrm{IU} / \mathrm{L}$ vs. $4.818 \pm 0.022 \mathrm{IU} / \mathrm{L}, P<0.0001)$. Regarding the $\mathrm{hCG}$ dose for triggering ovulation, PCOS patients required a significantly lower dose than control patients (mean \pm SE: $6130.95 \pm$ $29.977 \mathrm{IU}$ vs. $6868.69 \pm 12.752 \mathrm{IU}, P=0.0009$ ).
To confirm the CCs cDNA samples, we analyzed the mRNA level of ADAMTS1, which was reported to be expressed at a lower level in CCs derived from PCOS patients than control patients $[4,18]$. In our study, the PCOS group had lower ADAMTS1 expression in CCs than control patient CCs ( $0.0081 \pm 0.00011$ vs. $0.0104 \pm 0.00006, P<0.05)$ (Fig. $2 \mathrm{~A})$. In line with the inverse expression pattern of ADAMTS1 in mice, higher $H S P G 2$ expression was observed in the PCOS group $(0.0285 \pm 0.00034$ vs. $0.0172 \pm 0.00012, P<0.05)$ (Fig. 2B). Compared with the control group, we obtained more oocytes from the PCOS group $(17.739 \pm 0.183$ vs. $12.223 \pm 0.046, P<0.0001)$ (Fig. 2 C), who displayed significantly lower fertilization rates $(64.245 \pm 0.359 \%$ vs. $85.502 \pm$ $0.147 \%, P<0.0001$ ) (Fig. 2D). These results not only further validated the inverse expression pattern in human CCs and implied that CCs derived from PCOS patients had lower ADAMTS1 and higher HSPG 2 mRNA levels.

Because a significantly different hCG dose was used between PCOS and control patients, we verified whether hCG
Table 2 Comparison of the baseline information and $\mathrm{COH}$ outcomes between low and high ADAMTS1 expression groups in normal ovulatory women

\begin{tabular}{lccc}
\hline Variable & $\begin{array}{l}\text { Group with low ADAMTS1 } \\
\text { level }(n=53)\end{array}$ & $\begin{array}{l}\text { Group with high ADAMTS1 } \\
\text { level }(n=52)\end{array}$ & $P$ value \\
\hline Age (years) & $29.596 \pm 0.072$ & $29.490 \pm 0.063$ & NS \\
BMI (Kg/m $\left.{ }^{2}\right)$ & $22.104 \pm 0.051$ & $21.441 \pm 0.047$ & NS \\
Basal FSH (IU/L) & $6.575 \pm 0.038$ & $5.955 \pm 0.042$ & NS \\
LH (IU/L) & $4.089 \pm 0.144$ & $4.081 \pm 0.057$ & NS \\
Serum AMH (ng/mL) & $4.507 \pm 0.041$ & $5.104 \pm 0.047$ & NS \\
hCG dose (IU) & $6833.333 \pm 24.729$ & $6906.250 \pm 26.296$ & NS \\
No. of oocytes retrieved & $10.981 \pm 0.074$ & $13.490 \pm 0.102$ & 0.0072 \\
No. of matured (MII) oocytes & $10.692 \pm 0.073$ & $13.000 \pm 0.101$ & 0.0335 \\
No. of transplantable embryos & $5.385 \pm 0.059$ & $7.137 \pm 0.077$ & 0.0137 \\
No. of good quality embryos ${ }^{\#}$ & $2.462 \pm 0.037$ & $3.863 \pm 0.053$ & 0.0097 \\
Transplantable rate $(\%)$ & $72.872 \pm 0.510$ & $81.188 \pm 0.405$ & NS \\
& & & $(0.0824)$ \\
Good quality embryos rate $(\%)$ & $33.360 \pm 0.468$ & $42.469 \pm 0.448$ & NS \\
& & & $(0.055)$ \\
\hline
\end{tabular}

Continuous variables are expressed as mean \pm SE. NS, no significance; \#, non-parametric tests were used 
Table 3 Comparison of the baseline information and $\mathrm{COH}$ outcomes between low and high $H S P G 2$ expression groups in normal ovulatory women

\begin{tabular}{lccc}
\hline Variable & $\begin{array}{l}\text { Group with low HSPG2 } \\
\text { level (n=53) }\end{array}$ & $\begin{array}{l}\text { Group with high HSPG2 } \\
\text { level }(\mathrm{n}=52)\end{array}$ & $P$ value \\
\hline Age (years) & $29.173 \pm 0.064$ & $29.922 \pm 0.071$ & $\mathrm{NS}$ \\
BMI (Kg/m $\left.{ }^{2}\right)$ & $21.608 \pm 0.050$ & $21.9401 \pm 0.050$ & $\mathrm{NS}$ \\
Basal FSH (IU/L) & $6.158 \pm 0.039$ & $6.402 \pm 0.042$ & $\mathrm{NS}$ \\
LH (IU/L) & $5.244 \pm 0.135$ & $4.745 \pm 0.077$ & $\mathrm{NS}$ \\
Serum AMH (ng/mL) & $5.129 \pm 0.042$ & $4.507 \pm 0.046$ & $\mathrm{NS}$ \\
hCG dose (IU) & $6969.388 \pm 22.521$ & $6770.000 \pm 28.089$ & $\mathrm{NS}$ \\
No. of oocytes retrieved & $12.423 \pm 0.092$ & $12.020 \pm 0.092$ & $\mathrm{NS}$ \\
No. of matured (MII) oocytes & $12.192 \pm 0.091$ & $11.471 \pm 0.089$ & 0.0474 \\
No. of transplantable embryos & $6.962 \pm 0.067$ & $5.529 \pm 2.471$ & 0.045 \\
No. of good quality embryos & $3.827 \pm 0.048$ & $2.471 \pm 0.044$ & 0.0047 \\
Transplantable rate $(\%)$ & $85.217 \pm 0.311$ & $68.600 \pm 0.545$ & 0.0004 \\
Good quality embryos rate $(\%)$ & $45.850 \pm 0.444$ & $29.734 \pm 0.436$ & 0.0005 \\
\hline
\end{tabular}

Continuous variables are expressed as mean $\pm \mathrm{SE}$ dose affected ADAMTS1 and HSPG2 expression. A subanalysis for patients with similar hCG was performed in each group. PCOS patients were divided into two groups according to the hCG dose lower or higher than the median value (hCG $\leq 5000$ IU or hCG $>5000 \mathrm{IU}$ ). The normal ovulatory group was also divided into two groups according to the $\mathrm{hCG}$ dose lower or higher than the median value ( $\mathrm{hCG} \leq 7000 \mathrm{IU}$ or hCG $>7000 \mathrm{IU})$. We did not find any difference in ADAMTS1 and $H S P G 2$ expression, retrieved oocytes number, and fertilization rate between PCOS and control patients (Supplementary a

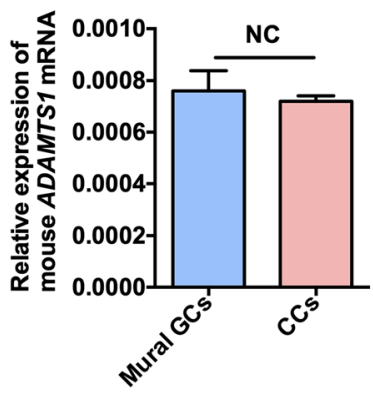

b

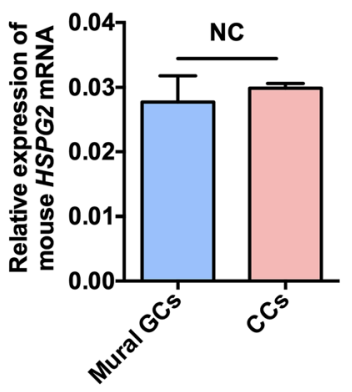

C

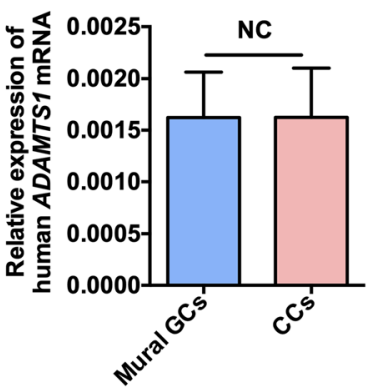

d

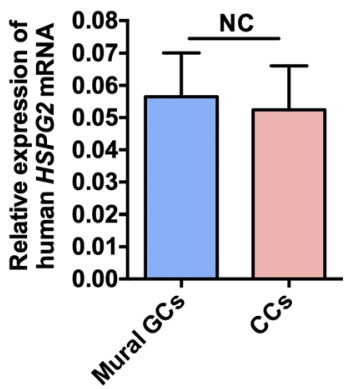

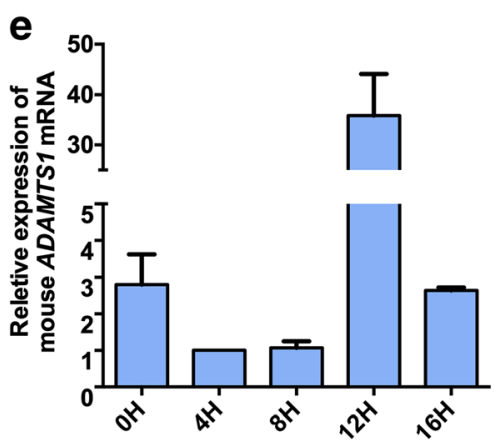

Fig. 1 The dynamic mRNA expression of ADAMTS1 and HSPG2 during ovulation. (A-B) RT-PCR results show the relative mRNA expression of ADAMTS1 and HSPG2 in cumulus cells and mural granulosa cells from mice. (C-D) RT-PCR results show the relative mRNA expression of ADAMTS1 and HSPG2 in cumulus cells and mural granulosa cells from patients. (E-F) The relative mRNA expression of ADAMTS1 and HSPG2
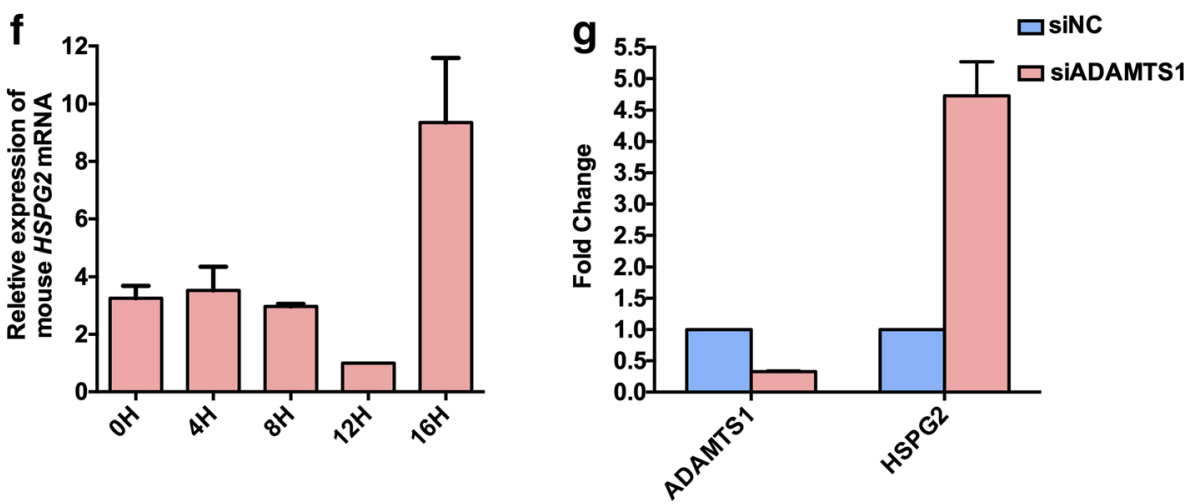

in cumulus cells at different timepoints $(0,4,8,12$, and $16 \mathrm{~h})$ after hCG followed by PMSG treatment for $48 \mathrm{~h}$. (G) RT-PCR analysis of the relative expression of $H S P G 2$ and ADAMTS1 in cumulus cells after ADAMTS1 RNAi treatment for $40 \mathrm{~h}$. Cumulus cells were collected from 10 COCs after IVM with a negative control (NC) or ADAMTS1 siRNAs treatment 

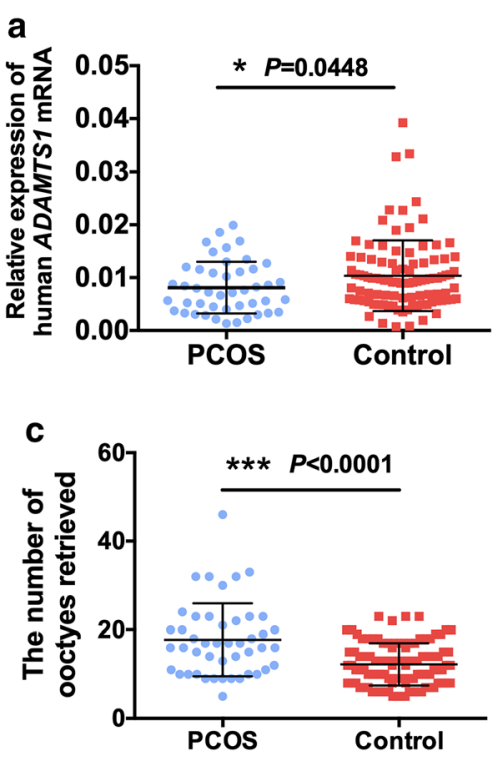

Fig. 2 Comparison of ADAMTS1 and HSPG2 mRNA levels in cumulus cells from PCOS and normal ovulatory control women. (A-B) RT-PCR results show the relative mRNA levels of ADAMTS1 (A) and HSPG2 (B) in the PCOS and control groups. (C) The number of oocytes retrieved from PCOS patients and normal ovulatory women. (D) The fertilization

Tables 2, 3). This suggested the dose of hCG did not affect the expression of ADAMTS1 and HSPG2.

\section{Relationship between ADAMTS1 or HSPG2 mRNA levels and oocyte developmental competence in normal ovulatory patients}

Next, we investigated whether the ADAMTS1 and HSPG2 expression levels were associated with oocyte developmental competence. Thus, normal ovulatory patients were recruited and divided into two groups according to whether the ADAMTS1 or HSPG2 expression levels in their CCs were higher or lower than the median value of all patients (higher, $n=52$; lower, $n=51$ ). Basal characteristics (including age, BMI, basal FSH, LH, serum AMH, and hCG dose) of the paired groups divided by the ADAMTS1 or HSPG2 expression levels were comparable, as shown in Tables 2 and 3, which reduced the likelihood of confounding bias.

The retrieved oocyte number and matured (MII) oocyte number in patients with high ADAMTS1 levels were significantly higher than that in patients with a low ADAMTS1 mRNA level $(13.490 \pm 0.102$ vs. $10.981 \pm 0.074, P=0.0072$; $13.000 \pm 0.101$ vs. $10.692 \pm 0.073, P=0.0335$ ) (Table 2). Regarding day 3 embryos, more transferable embryos ( $7.137 \pm 0.077$ vs. $5.385 \pm 0.059, P=0.0137)$ (Table 2) and more good quality embryos $(3.863 \pm 0.053$ vs. $2.462 \pm$ $0.037, P=0.0097$ ) (Table 2) were obtained from the high ADAMTS1 group compared with the low ADAMTS1 group (Table 2). Although a higher transferable rate and good quality embryo rate were observed in the high ADAMTS1 group
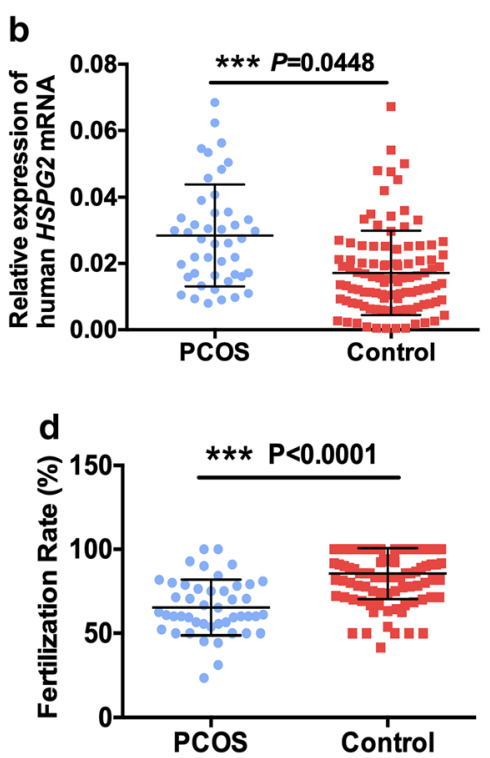

rate was compared between the PCOS and control groups. Comparisons of the outcome with ADAMTS1 and HSPG2 levels in PCOS and control groups were performed using the Student's two-tailed $t$-test or nonparametric test. Bars indicate standard deviation of the mean of all subjects in the group: $* P<0.05 ; * * * P<0.001$

compared with the low ADAMTS1 group, this did not reach statistical significance $(81.188 \pm 0.405$ vs. $72.872 \pm 0.510$, $P=0.0824 ; 42.469 \pm 0.448$ vs. $33.360 \pm 0.468, P=0.055)$ (Table 2). These results suggested that ADAMTS1 levels were linked with oocyte and embryo number and quality.

Regarding HSPG2, although similar oocyte numbers were retrieved from the low and high $H S P G 2$ groups (12.423 \pm 0.092 vs. $12.020 \pm 0.092$, NS) (Table 3), more mature MII oocytes $(12.192 \pm 0.091$ vs. $11.471 \pm 0.089, P=0.0474)$ (Table 3), more transferable embryos $(6.962 \pm 0.067$ vs. $5.529 \pm 2.471, P=0.045$ ) (Table 3 ), and more good quality embryos $(3.827 \pm 0.048$ vs. $2.471 \pm 0.044, P=0.0047)$ (Table 3) were obtained from the low HSPG2 group than from the high $H S P G 2$ group. Moreover, the transferable embryo rate and good quality embryo rate are valuable characteristics to evaluate oocyte developmental competence. The transferable rate and good quality embryo rate were significantly higher $(85.217 \pm 0.311 \%$ vs. $68.600 \pm 0.545 \%, P=0.004$; $45.850 \pm 0.444 \%$ vs. $29.734 \pm 0.436 \%, P=0.0005$ ) in patients with lower HSPG2 mRNA levels (Table 3). These results indicated $H S P G 2$ was closely associated with oocyte quality and developmental competence. Overall, the high ADAMTS1 and low HSPG2 expression levels were associated with better oocyte developmental competence.

\section{The predictive power of ADAMTS1 and HSPG2 mRNA levels with pregnancy outcome}

ROC analysis was performed and the area under the curve (AUC) was used to estimate the accuracy of potential 
biomarkers. To investigate the predictive effect of the relative mRNA expression of ADAMTS1 and HSPG2 on COH outcome, implantation was evaluated after the IVF/ICSI procedure and embryo transfer.

Implantation was determined by serum hCG levels measured at 14 days after embryo transfer. When we analyzed the correlation of ADAMTS1 or HSPG2 mRNA level with implantation separately, there was no statistical significance (ADAMTS1: $\mathrm{AUC}=0.512, P=0.837 ;$ HSPG2: $\mathrm{AUC}=0.488$, $P=0.837$ ) (Fig. 3A-B). Interestingly, the AUC of ADAMTS1 and $H S P G 2$ to predict pregnancy was 0.738 . Further calculation showed that the positive predictive value was $79.3 \%$, and the negative predictive value was $55.6 \%$ (Fig. 3C). Therefore, we concluded that the co-measurement of ADAMTS1 and $H S P G 2$ assists in the prediction of pregnancy outcomes.

\section{Discussion}

In clinical situations, evaluating oocyte quality for IVF/ICSI and choosing which embryo to transplant are vital considerations for $\mathrm{COH}$ women. Previous studies have shown that ADAMTS1, whose expression is LH/hCG and follicle-size dependent, and which shows rapidly induced expression $(>20-$ fold increase in CCs after hCG treatment in humans) [42, 43], was related to follicular development and ovulation and may be involved in female infertility such as PCOS [3, 7, 8, 44, 45]. PCOS patients have lower ADAMTS1 levels in CCs and higher ADAMTS1 levels in follicular fluids compared with normal ovulatory women $[4,25,26]$. A recent study showed that miR-375 regulated COC maturation by targeting ADAMTS1 [46]. However, whether ADAMTS1 is a predictor of oocyte and embryo quality in normal ovulatory patients is unclear. In addition, analysis of $\mathrm{t}$ a single molecule is inadequate and inaccurate to judge oocyte competence. Although previous studies have shown the co-analysis of ADAMTS1 and versican improved accuracy [14, 20, 22], our study investigated the use of a new molecule to predict high quality oocytes.

Proteoglycans consist of a protein core and covalently attached glycosaminoglycan (GAG) chains, which are basic components of the ECM [13]. Proteoglycans such as versican, aggrecan, perlecan, laminin, prolargin, and collagen type IV exist in ovarian follicles [26]. Versican and aggrecan, but not perlecan, are confirmed substrates of ADAMTS1 [25]. There is limited evidence for the downregulation of $H S P G 2$ expression at the mRNA level by ADAMTS1. However, studies reported that perlecan in follicular fluids reached a peak at $12 \mathrm{~h}$ after injecting hCG [47] and may have a close relationship with oocyte developmental competence [48-50]. Because perlecan in follicular fluids is derived from GCs, it will be interesting to investigate correlations between HSPG2 mRNA levels in human CCs and oocyte developmental competence. This study focused on both PCOS patients and normal ovulatory $\mathrm{COH}$ women, and we found that patients with lower HSPG2 mRNA levels in $\mathrm{CCs}$ had better $\mathrm{COH}$ outcomes.

To date, most studies have focused on the pre-embryo transfer period, including the mature oocyte and fertilization rate, but no study has examined the predictive power of ADAMTS1/HSPG2 mRNA levels in human CCs with oocyte quality, especially during clinical pregnancy. This study extended the follow-up time to implantation.

We compared the relative expression of ADAMTS1 in human CCs between PCOS and normal ovulatory women and found lower ADAMTS1 expression in PCOS as previously reported [4]. These findings also confirm a study by Brown et al. that reported ovulation and subsequent fertilization were seriously impaired in ADAMTS1-deficient mice [14]. At the same time, we found that HSPG2 mRNA levels were a

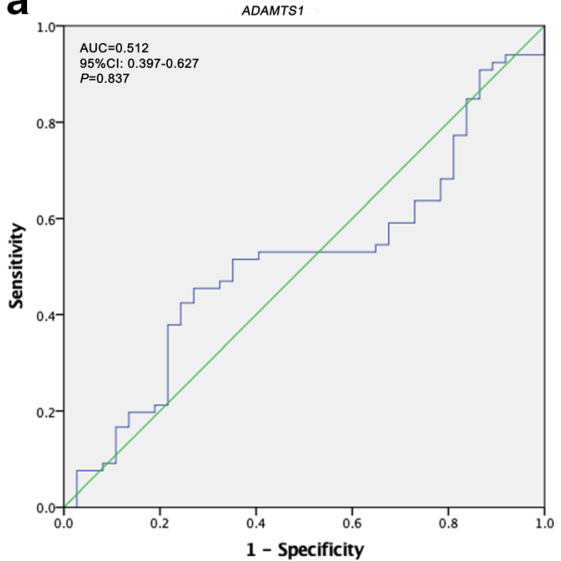

b

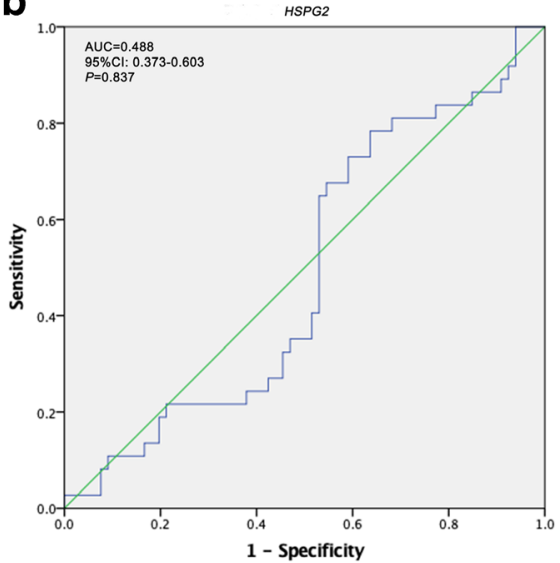

C

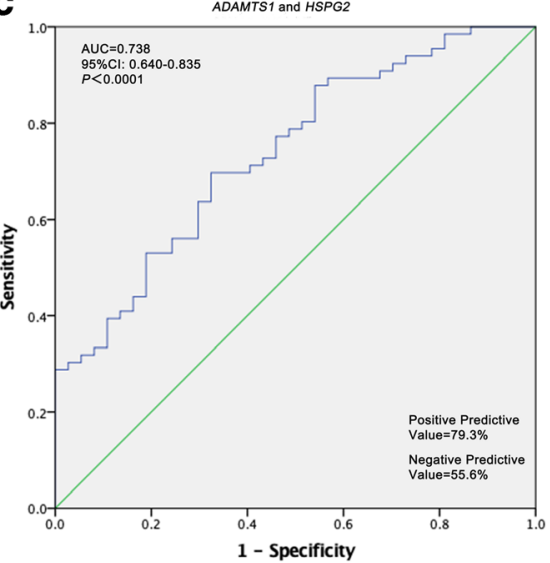

Fig. 3 ROC curve of ADAMTS1/HSPG2 mRNA levels and successful $\mathrm{COH}$ implantation outcomes. (A) ROC curve of ADAMTS1 mRNA levels and successful $\mathrm{COH}$ implantation outcomes. (B) ROC curve of $H S P G 2$ mRNA levels and successful $\mathrm{COH}$ implantation outcomes. (C)
ROC curve of co-measurement of ADAMTS1/HSPG2 mRNA levels and successful COH implantation outcomes; AUC, area under curve; 95\% CI, $95 \%$ confidence interval 
significantly higher in CCs from PCOS patients than in control patients. After $\mathrm{COH}$, the PCOS group had low ADAMTS1 and high $H S P G 2$ levels with a significantly low fertilization rate, although more oocytes were retrieved for pregnancy from PCOS patients.

During ovulation in mice, we found that the expression of ADAMTS1 or HSPG2 mRNA were comparable between mural and cumulus cells derived from mice or humans around the time of ovulation. Therefore, in this study, easily harvested CCs were used to evaluate the expression of ADAMTS1 and $H S P G 2$. Of note, the levels of ADAMTS1 and HSPG2 were inversely correlated, especially at hCG $12 \mathrm{~h}$ in C57BL/6 mice, a timepoint near ovulation. Moreover, HSPG2 was significantly increased after downregulating ADAMTS1. Therefore, we speculate that ADAMTS1 and HSPG2 may have related functions during ovulation.

To date, no studies have reported a relationship between ADAMTS1 and HSPG 2 expression and $\mathrm{COH}$ outcomes in normal ovulatory women. Therefore, we detected the mRNA levels of ADAMTS1 and HSPG2 in tubal or male factor patients. Patients were divided into two groups based on ADAMTS1 or $H S P G 2$ expression levels higher or lower than the median value (low and high), to analyze the relationship between ADAMTS1 or $H S P G 2$ expression and $\mathrm{COH}$ outcomes. In tubal or male factor patients, the group with higher ADAMTS1 levels and lower $H S P G 2$ levels had a better clinical outcome, with more MII stage oocytes, transferable embryos, good quality embryos, and a higher rate of transferable embryos or good quality embryos. Our results indicate that the relative expression of ADAMTS1 was correlated positively with the number of oocytes and embryos, while the relative expression of $H S P G 2$ correlated positively with the quality of oocytes and embryos. Therefore, the co-measurement of ADAMTS1 and HSPG2 might better predict $\mathrm{COH}$ outcomes and later analysis demonstrated this.

ROC analysis was performed, and the AUC was used to estimate the accuracy of potential biomarkers, including ADAMTS1 mRNA with implantation, HSPG2 mRNA with implantation, and co-measurement of ADAMTS1 mRNA and HSPG 2 mRNA with implantation. Interestingly, the comeasurement of two genes improved the predictive power.

In this study, HSPG2 seemed more significant than ADAMTS1 at predicting oocyte and embryo quality. Because perlecan has a critical role in numerous physiological processes [22], the relative expression of HSPG2 mRNA may be more useful than that of ADAMTS1 to predict good quality embryos. For example, Princivalle et al. reported that perlecan provided attachment for anticoagulant heparan sulfate proteoglycans (aHSPGs) on the cell surface and in the extracellular matrix, which contributed to the control of proteolytic activity involved in follicular development and ovulation [51]. Abnormal $H S P G 2$ encodes too much or too little perlecan, which may influence the interaction with aHSPGs and ADAMTS1, while at the same time, it may affect endothelial vascular growth during follicular development [3, 8, 10, 24, 44].

Substantial evidence has shown that EGF-like factors (AREG, EREG, BTC), EGFR and the ERK1/ERK2 signaling cascade have critical roles for cumulus expansion, oocyte maturation, and follicle rupture. Many genes related to ovulation including AREG, EREG, HAS2, PTGS2, CITED2, CITED4, and ADAMTS1 were induced by LH via the ERK1/ERK2 signaling cascade $[6,52-54]$. These gene expressions may be essential for oocyte meiotic maturation. For example, the spindles of MII oocytes from AREG knockout mice were abnormal [55]. Previous studies have confirmed that the expression of some of these genes in GCs including AREG, EREG, and CITED2 might be potential biomarkers to predict human oocyte quality in IVF/ICSI [1, 23, 56-58].

And $H S P G 2 /$ perlecan is important for chondrocyte clustering and mediates its effect through the ERK pathway [59]. Based on these studies and our research, the ERK pathway may be involved in the mechanism of how ADAMTS1/HSPG2 affect oocyte quality. Co-measurement of ADAMTS1/HSPG2 mRNAs in human CCs might be a predictive method for human oocyte quality. We are interested in exploring further the correlation between the mRNA levels of these genes in single denuded MII oocytes and clinical outcomes.

In conclusion, we shed light on the expression of ADAMTS1 and HSPG2 in mural GCs and CCs in mice and humans and found that high ADAMTS1 levels and low $H S P G 2$ levels in CCs were associated with high oocyte quality and $\mathrm{COH}$ outcomes in both PCOS and normal ovulatory women. Co-measurement of ADAMTS1 and HSPG2 improved the predictive power to estimate clinical pregnancy outcomes. Detection of ADAMTS1 and HSPG2 mRNAs in human CCs may help doctors make decisions in specific cases, such as patients who have failed multiple transplants. However, prior to use for clinical diagnosis, evidence to validate the close correlation between ADAMTS1 and HSPG2 mRNA levels and pregnancy outcomes (such as implantation rate and live birth rate) in other or larger populations is required. This study only identified the correlation between ADAMTS1 and HSPG2 expression levels and $\mathrm{COH}$ outcomes. Whether low ADAMTS1 expression is the cause of anovulation in PCOS patients needs to be investigated further. Additional studies are also needed to clarify how ADAMTS1 influences the expression of $H S P G 2$.

Acknowledgments We thank the ART team of Sir Run Run Shaw Hospital for the support of this study.

Authors' contribution Yerong Ma: Sample collection, data collection, data analysis, and manuscript writing.

Jiamin Jin: Sample collection, data collection, data analysis.

Xiaomei Tong: Sample collection, manuscript editing, funding acquisition.

Weijie Yang: Project development, data analysis. 
Peipei Ren: Data collection.

Yongdong Dai: Project development.

Yibin Pan: Project development.

YinLi Zhang: Project development, manuscript editing, funding acquisition

Songying Zhang: Project development, manuscript editing, funding acquisition.

Funding information And this study is funded by the Key Research and Development Program of Zhejiang Province (2017C03022), National Natural Science Foundation of China (31701260, 81401264), and Zhejiang Province Medical Science and Technology Plan Project (2017178803)

\section{Compliance with ethical standards}

Conflict of interest The authors declare that they have no conflict(s) of interest.

Open Access This article is licensed under a Creative Commons Attribution 4.0 International License, which permits use, sharing, adaptation, distribution and reproduction in any medium or format, as long as you give appropriate credit to the original author(s) and the source, provide a link to the Creative Commons licence, and indicate if changes were made. The images or other third party material in this article are included in the article's Creative Commons licence, unless indicated otherwise in a credit line to the material. If material is not included in the article's Creative Commons licence and your intended use is not permitted by statutory regulation or exceeds the permitted use, you will need to obtain permission directly from the copyright holder. To view a copy of this licence, visit http://creativecommons.org/licenses/by/4.0/.

\section{References}

1. Yamashita Y, Hishinuma M, Shimada M. Activation of PKA, p38 MAPK and ERK1/2 by gonadotropins in cumulus cells is critical for induction of EGF-like factor and TACE/ADAM17 gene expression during in vitro maturation of porcine COCs. J Ovarian Res. 2009;2:20.

2. Richards JS, Hernandez-Gonzalez I, Gonzalez-Robayna I, Teuling E, Lo Y, Boerboom D, et al. Regulated expression of ADAMTS family members in follicles and cumulus oocyte complexes: evidence for specific and redundant patterns during ovulation. Biol Reprod. 2005;72(5):1241-55.

3. Shozu M, Minami N, Yokoyama $H$, Inoue $M$, Kurihara $H$, Matsushima K, et al. ADAMTS-1 is involved in normal follicular development, ovulatory process and organization of the medullary vascular network in the ovary. J Mol Endocrinol. 2005;35(2):34355.

4. Xiao S, et al. Evidence for decreased expression of ADAMTS-1 associated with impaired oocyte quality in PCOS patients. J Clin Endocrinol Metab. 2014;99(6):E1015-21.

5. Tsubota K, Kanki M, Noto T, Nakatsuji S, Oishi Y, Matsumoto M, et al. Altered gene expression profile in ovarian follicle in rats treated with indomethacin and RU486. J Toxicol Sci. 2015;40(3): 413-25.

6. Schuermann Y, Rovani MT, Gasperin B, Ferreira R, Ferst J, Madogwe E, et al. ERK1/2-dependent gene expression in the bovine ovulating follicle. Sci Rep. 2018;8(1):16170.

7. GohariTaban S, Amiri I, Soleimani Asl S, Saidijam M, Yavangi M, Khanlarzadeh E, et al. Abnormal expressions of ADAMTS-1,
ADAMTS-9 and progesterone receptors are associated with lower oocyte maturation in women with polycystic ovary syndrome. Arch Gynecol Obstet. 2019;299(1):277-86.

8. Rodgers RJ, Irving-Rodgers HF, Russell DL. Extracellular matrix of the developing ovarian follicle. Reproduction. 2003;126(4):41524.

9. Irving-Rodgers HF, Harland ML, Rodgers RJ. A novel basal lamina matrix of the stratified epithelium of the ovarian follicle. Matrix Biol. 2004;23(4):207-17.

10. Brown HM, Dunning KR, Robker RL, Pritchard M, Russell DL. Requirement for ADAMTS-1 in extracellular matrix remodeling during ovarian folliculogenesis and lymphangiogenesis. Dev Biol. 2006;300(2):699-709.

11. Irving-Rodgers HF, Catanzariti KD, Aspden WJ, D'Occhio MJ, Rodgers RJ. Remodeling of extracellular matrix at ovulation of the bovine ovarian follicle. Mol Reprod Dev. 2006;73(10):1292302.

12. Irving-Rodgers HF, Harland ML, Sullivan TR, Rodgers RJ. Studies of granulosa cell maturation in dominant and subordinate bovine follicles: novel extracellular matrix focimatrix is co-ordinately regulated with cholesterol side-chain cleavage CYP11A1. Reproduction. 2009;137(5):825-34.

13. Chen CP, Liu SH, Lee MY, Chen YY. Heparan sulfate proteoglycans in the basement membranes of the human placenta and decidua. Placenta. 2008;29(4):309-16.

14. Brown HM, Dunning KR, Robker RL, Boerboom D, Pritchard M, Lane M, et al. ADAMTS1 cleavage of versican mediates essential structural remodeling of the ovarian follicle and cumulus-oocyte matrix during ovulation in mice. Biol Reprod. 2010;83(4):549-57.

15. Nagashima T, Kim J, Li Q, Lydon JP, DeMayo F, Lyons KM, et al. Connective tissue growth factor is required for normal follicle development and ovulation. Mol Endocrinol. 2011;25(10):1740-59.

16. Norambuena-Soto I, Núñez-Soto C, Sanhueza-Olivares F, CancinoArenas N, Mondaca-Ruff D, Vivar R, et al. Transforming growth factor-beta and Forkhead box $\mathrm{O}$ transcription factors as cardiac fibroblast regulators. Biosci Trends. 2017;11(2):154-62.

17. Camaioni A, et al. Proteoglycans and proteins in the extracellular matrix of mouse cumulus cell-oocyte complexes. Arch Biochem Biophys. 1996;325(2):190-8.

18. Russell DL, Doyle KM, Ochsner SA, Sandy JD, Richards JS Processing and localization of ADAMTS-1 and proteolytic cleavage of versican during cumulus matrix expansion and ovulation. $\mathrm{J}$ Biol Chem. 2003;278(43):42330-9.

19. Malgouries S, Thibaut S, Bernard BA. Proteoglycan expression patterns in human hair follicle. Br J Dermatol. 2008;158(2):234-42.

20. Curry TE Jr. ADAMTS1 and versican: partners in ovulation and fertilization. Biol Reprod. 2010;83(4):505-6.

21. Hatzirodos N, Nigro J, Irving-Rodgers HF, Vashi AV, Hummitzsch $\mathrm{K}$, Caterson B, et al. Glycomic analyses of ovarian follicles during development and atresia. Matrix Biol. 2012;31(1):45-56.

22. Vasconcelos GL, et al. Effects of growth differentiation factor- 9 and FSH on in vitro development, viability and mRNA expression in bovine preantral follicles. Reprod Fertil Dev. 2013;25(8):1194-203.

23. Fauser BC, et al. Consensus on women's health aspects of polycystic ovary syndrome (PCOS): the Amsterdam ESHRE/ASRMSponsored 3rd PCOS Consensus Workshop Group. Fertil Steril. 2012;97(1):28-38 e25.

24. Ambekar AS, et al. Proteomics of follicular fluid from women with polycystic ovary syndrome suggests molecular defects in follicular development. J Clin Endocrinol Metab. 2015;100(2):744-53.

25. Karakose $M$, et al. Clinical significance of ADAMTS1, ADAMTS5, ADAMTS9 aggrecanases and IL-17A, IL-23, IL-33 cytokines in polycystic ovary syndrome. J Endocrinol Investig. 2016;39(11):1269-75. 
26. Tola EN, Karatopuk DU, Koroglu N, Ergin M, Oral HB. Follicular ADAMTS-1 and aggrecan levels in polycystic ovary syndrome. J Assist Reprod Genet. 2017;34(6):811-6.

27. Winkler S, Stahl RC, Carey DJ, Bansal R. Syndecan-3 and perlecan are differentially expressed by progenitors and mature oligodendrocytes and accumulate in the extracellular matrix. J Neurosci Res. 2002;69(4):477-87.

28. Datta MW, et al. Perlecan, a candidate gene for the CAPB locus, regulates prostate cancer cell growth via the sonic hedgehog pathway. Mol Cancer. 2006;5:9.

29. Miller JD, Stevens ET, Smith DR, Wight TN, Wrenshall LE. Perlecan: a major IL-2-binding proteoglycan in murine spleen. Immunol Cell Biol. 2008;86(2):192-9.

30. Bollineni JS, Alluru I, Reddi AS. Heparan sulfate proteoglycan synthesis and its expression are decreased in the retina of diabetic rats. Curr Eye Res. 1997;16(2):127-30.

31. Smith S, Hassell JR. Focus on molecules: perlecan (HSPG2). Exp Eye Res. 2006;83(3):471-2.

32. Gustafsson E, Almonte-Becerril M, Bloch W, Costell M. Perlecan maintains microvessel integrity in vivo and modulates their formation in vitro. PLoS One. 2013;8(1):e53715.

33. Farach-Carson MC, Warren CR, Harrington DA, Carson DD. Border patrol: insights into the unique role of perlecan/heparan sulfate proteoglycan 2 at cell and tissue borders. Matrix Biol. 2014;34:64-79.

34. Murdoch AD, Dodge GR, Cohen I, Tuan RS, Iozzo RV. Primary structure of the human heparan sulfate proteoglycan from basement membrane (HSPG2/perlecan). A chimeric molecule with multiple domains homologous to the low density lipoprotein receptor, laminin, neural cell adhesion molecules, and epidermal growth factor. J Biol Chem. 1992;267(12):8544-57.

35. Mishra M, Naik VV, Kale AD, Ankola AV, Pilli GS. Perlecan (basement membrane heparan sulfate proteoglycan) and its role in oral malignancies: an overview. Indian J Dent Res. 2011;22(6): 823-6.

36. Jedrzejuk D, et al. Association of serum glypican-4 levels with cardiovascular risk predictors in women with polycystic ovary syndrome - a pilot study. Gynecol Endocrinol. 2016;32(3):223-6.

37. Grindel BJ, et al. Matrilysin/matrix metalloproteinase-7(MMP7) cleavage of perlecan/HSPG2 creates a molecular switch to alter prostate cancer cell behavior. Matrix Biol. 2014;36:64-76.

38. Bayasula, et al. A proteomic analysis of human follicular fluid: comparison between fertilized oocytes and non-fertilized oocytes in the same patient. J Assist Reprod Genet. 2013;30(9):1231-8.

39. Revised 2003. Consensus on diagnostic criteria and long-term health risks related to polycystic ovary syndrome (PCOS). Hum Reprod. 2004;19(1):41-7.

40. Lv Y, Zhao SG, Lu G, Leung CK, Xiong ZQ, Su XW, et al. Identification of reference genes for qRT-PCR in granulosa cells of healthy women and polycystic ovarian syndrome patients. Sci Rep. 2017;7(1):6961.

41. Howles CM. Textbook of assisted reproductive techniques. Book. 2013;2(2):70.

42. Yung Y, Maman E, Konopnicki S, Cohen B, Brengauz M, Lojkin I, et al. ADAMTS-1: a new human ovulatory gene and a cumulus marker for fertilization capacity. Mol Cell Endocrinol. 2010;328(1-2):104-8.

43. Wissing ML, Kristensen SG, Andersen CY, Mikkelsen AL, Høst T, Borup R, et al. Identification of new ovulation-related genes in humans by comparing the transcriptome of granulosa cells before and after ovulation triggering in the same controlled ovarian stimulation cycle. Hum Reprod. 2014;29(5):997-1010.

44. Matti N, Irving-Rodgers HF, Hatzirodos N, Sullivan TR, Rodgers RJ. Differential expression of focimatrix and steroidogenic enzymes before size deviation during waves of follicular development in bovine ovarian follicles. Mol Cell Endocrinol. 2010;321(2): 207-14.

45. Vabre P, Gatimel N, Moreau J, Gayrard V, Picard-Hagen N, Parinaud J, et al. Environmental pollutants, a possible etiology for premature ovarian insufficiency: a narrative review of animal and human data. Environ Health. 2017;16(1):37.

46. Zhang J, et al. MicroRNA-375 regulates oocyte in vitro maturation by targeting ADAMTS1 and PGR in bovine cumulus cells. Biomed Pharmacother. 2019;118:109350.

47. Poulsen LC, et al. Progressive changes in human follicular fluid composition over the course of ovulation: quantitative proteomic analyses. Mol Cell Endocrinol. 2019;495:110522.

48. Dunning KR, Akison LK, Russell DL, Norman RJ, Robker RL. Increased beta-oxidation and improved oocyte developmental competence in response to 1-carnitine during ovarian in vitro follicle development in mice. Biol Reprod. 2011;85(3):548-55.

49. O'Gorman A, Wallace M, Cottell E, Gibney MJ, McAuliffe F, Wingfield $\mathrm{M}$, et al. Metabolic profiling of human follicular fluid identifies potential biomarkers of oocyte developmental competence. Reproduction. 2013;146(4):389-95.

50. Dumesic DA, Meldrum DR, Katz-Jaffe MG, Krisher RL, Schoolcraft WB. Oocyte environment: follicular fluid and cumulus cells are critical for oocyte health. Fertil Steril. 2015;103(2):30316.

51. Princivalle M, Hasan S, Hosseini G, de Agostini AI. Anticoagulant heparan sulfate proteoglycans expression in the rat ovary peaks in preovulatory granulosa cells. Glycobiology. 2001;11(3):183-94.

52. Fan HY, Liu Z, Shimada M, Sterneck E, Johnson PF, Hedrick SM, et al. MAPK3/1 (ERK1/2) in ovarian granulosa cells are essential for female fertility. Science. 2009;324(5929):938-41.

53. Sayasith K, Lussier J, Sirois J. Molecular characterization and transcriptional regulation of a disintegrin and metalloproteinase with thrombospondin motif 1 (ADAMTS1) in bovine preovulatory follicles. Endocrinology. 2013;154(8):2857-69.

54. Zhang YL, Xia Y, Yu C, Richards JS, Liu J, Fan HY. CBP-CITED4 is required for luteinizing hormone-triggered target gene expression during ovulation. Mol Hum Reprod. 2014;20(9):850-60.

55. Chen J, Torcia S, Xie F, Lin CJ, Cakmak H, Franciosi F, et al. Somatic cells regulate maternal mRNA translation and developmental competence of mouse oocytes. Nat Cell Biol. 2013;15(12):1415-23.

56. Ouandaogo ZG, Frydman N, Hesters L, Assou S, Haouzi D, Dechaud H, et al. Differences in transcriptomic profiles of human cumulus cells isolated from oocytes at GV, MI and MII stages after in vivo and in vitro oocyte maturation. Hum Reprod. 2012;27(8): 2438-47.

57. Guzman L, Adriaenssens T, Ortega-Hrepich C, Albuz FK, Mateizel I, Devroey P, et al. Human antral follicles $<6 \mathrm{~mm}$ : a comparison between in vivo maturation and in vitro maturation in non-hCG primed cycles using cumulus cell gene expression. Mol Hum Reprod. 2013;19(1):7-16.

58. Fang Y, et al. Cited2 protein level in cumulus cells is a biomarker for human embryo quality and pregnancy outcome in one in vitro fertilization cycle. Fertil Steril. 2016;105(5):1351-1359.e4.

59. Martinez J R, Grindel B J, Hubka K M, et al. Perlecan/HSPG2: Signaling role of domain IV in chondrocyte clustering with implications for Schwartz-Jampel Syndrome[J]. Journal of cellular biochemistry, 2019;120(2): 2138-2150.

Publisher's note Springer Nature remains neutral with regard to jurisdictional claims in published maps and institutional affiliations. 